\title{
Importance of Diet in Inflammatory Bowel Disease - Data from the Patients' View
}

\author{
Tamas Molnar ${ }^{1 *}$, Klaudia Farkas ${ }^{1}$, Ferenc Nagy ${ }^{1}$, Monika Szucs ${ }^{2}$, Anita Balint ${ }^{1}$, Zoltan Szepes ${ }^{1}$, Noemi Vass ${ }^{3}$, and Tibor \\ Wittmann $^{1}$ \\ ${ }^{1}$ First Department of Medicine, University of Szeged, Szeged, Hungary \\ ${ }^{2}$ Department of Medical Physics and Informatics, University of Szeged, Szeged, Hungary \\ ${ }^{3}$ Department of Pediatrics and Pediatric Health Care Center, University of Szeged, Szeged, Hungary
}

Received: August 30, 2014; Accepted: October 05, 2014; Published: October 17, 2014

*Corresponding author: Tamas Molnar, First Department of Medicine, University of Szeged, H-6720. Koranyi fasor 8. Szeged, Hungary, Fax: 0036/62455-185; E-mail: molnar.tamas@med.u-szeged.hu

\begin{abstract}
Background: Inflammatory Bowel Diseases (IBD; Crohn's Disease [CD] and Ulcerative Colitis [UC]) are chronic, relapsing conditions caused by genetic, immunologic and environmental factors. However, the role of diet is controversial in the management of IBD.

Objective: The aim of this study was to assess our patients opinion about the importance of diet and to evaluate the role of special meals and ingredients in the relapse of IBD symptoms using a structured questionnaire.

Patients and method: One hundred and fifty-four patients participated in this nutritional survey. Most of the patients were female (106 vs. 48), mean disease duration was 11.8 years and the two types of IBD were represented almost equally (CD 54.7\%, UC 45.3\%).

Results: $42.3 \%$ of CD patients vs. $19.4 \%$ of UC patients thought that nutrition has an important role in the development of IBD. $39.6 \%$ of our patients are on diet permanently. $52.7 \%$ of IBD patients thought that diet can prevent relapse significantly more patients with CD compared to UC. $68.6 \%$ of the IBD patients felt that diet is as important as drug therapy. $91 \%$ of $\mathrm{CD}$ and $79 \%$ of UC patients reported ingredients or meals which worsen the disease activity symptoms.
\end{abstract}

Conclusions: On the basis of our survey, diet is more important in the pathogenesis and management of CD than UC. Most of the patients think that diet can prevent relapse and more than two-thirds of the patients find nutrition to be as important as drug therapy. We should pay more attention to the nutritional factors during the management of IBD.

Keywords: Inflammatory bowel disease; Diet; Management

\section{Introduction}

Inflammatory Bowel Diseases (IBD; Crohn's Disease [CD] and Ulcerative Colitis [UC]) are chronic, relapsing conditions influenced by several environmental factors. Diet is widely one of the most frequently studied environmental factors in the development of IBD; however, its evidence in the relapses of IBD is still missing. Moreover, very little information is available on the patients' own perceptions about the role of diet in IBD, although according to some patients' beliefs, certain foods have been proved to worsen the symptoms of IBD [1].

Appropriate diet is supposed to reduce symptoms in inflammatory activity of IBD. According to the guidelines of the World Gastroenterology Organization 2010, in case of clinical activity, a decrease in the amount of fibres and a low-residue diet may be beneficial [2]. The American Dietetic Association [3], the world's largest organization of food and nutrition professionals, recommends a special diet for patients with active or inactive disease using their 'Recommended Foods chart'. On the other hand, according to the Global IBD Dietary Clinical Practice Guideline 'no special diet has been proven to be effective for preventing or treating CD symptoms or inflammation, but it is very important that people who have $\mathrm{CD}$ follow a nutritious diet, and avoid malnutrition, weight loss and any foods that seem to worsen symptoms. Symptoms caused by special food may be unique and can vary from person to person' [4].

Identifying dietary factors as predictors for development of IBD and the therapeutic efficacy of dietary modifications are becoming more important in the current management of the disease. The aim of this study was to assess our patients' beliefs about the importance of diet and to evaluate the role of special foods and ingredients in the relapse of IBD symptoms using a new, structured questionnaire. The most important questions of this study were the patients' opinion on the role of diet in the development of IBD, in disease course and relapse and in other special cases when diet has a crucial role.

\section{Patients and Methods}

\section{Patients}

This study was conducted between November 2013 and May 2014 at the First Department of Medicine, University of Szeged, using a self-administered questionnaire. Adults over the age of 18 years with an existing diagnosis of CD or UC for at least three months were included in the study. Overall, 154 outpatients 
treated at our tertiary IBD center participated in our survey. Most of the patients were female (106 vs. 48), and the two types of IBD were represented almost equally (CD 54.7\%, UC 45.3\%). The median age was 38 years (IQR, 29-53) and median disease duration was 9 years (IQR, 5-18). Average body mass index was 24.0 (IQR, 14.8-39.3). 50.6\% of the 154 patients were treated with aminosalycilates, $15.6 \%$ with methylprednisolone, $17.5 \%$ with budesonide, $50.6 \%$ with azathioprine, $1.3 \%$ with cyclosporine and $24.7 \%$ with anti TNF- $\alpha$ therapy. Table 1 summarizes the baseline demographic and clinical characteristics of the participants. The study was approved by the Regional and Institutional Human Medical Biological Research Ethics Committee of the University of Szeged.

\section{Questionnaire}

The questionnaires were conducted prior to the patients' medical consultation. We used a self-administered questionnaire (Attachment) to obtain information on demographic and clinical factors, dietary habits and their own opinion about the associations between dietary factors and disease course. The questionnaire based on literature data and our own experience containing relevant questions. No validation processes were applied. Participating physicians distributed and collected the questionnaires.

\section{Statistical methods}

The comparisons of categorical and continuous variables were accomplished using the chi-square test and Fisher's exact test. Logistic regression analysis was used to evaluate correlations between variables. $p<0.05$ was considered statistically significant.

\section{Results}

We evaluated whether the quality of eating is perceived by patients to be a risk factor for IBD: $30.5 \%$ of patients believed that certain foods could initiate the disease, and $50.6 \%$ believed that it can be a risk for relapse. $38.3 \%$ of the patients were

Table 1: Baseline demographic and clinical characteristics of the participants.

\begin{tabular}{|l|c|c|}
\hline & CD & UC \\
\hline Male number (\%) & 28.4 & 32.8 \\
\hline Present age (median, years) & 36 (IQR, 28-45) & 40.5 (IQR, 34-56) \\
\hline Disease duration (median, years) & 8 (IQR, 6-14) & 10 (IQR, 4.3-19) \\
\hline BMI (median) & $22.8(20.4-$ & 24.8 (IQR, 21.6- \\
& $25.3)$ & $27.2)$ \\
\hline Therapy (\%) & & \\
- Aminosalycilate & 39.5 & 61.2 \\
- $\quad 16$ & 16.4 \\
- $\quad$ Budesylprednisolone & 16 & 19.4 \\
- $\quad$ Azathioprine & 53.1 & 1.5 \\
- Cyclosporine & 1.2 & 20.9 \\
\hline Anti TNF- $\alpha$ & 29.6 & 13.4 \\
\hline Lactose intolerance (\%) & 14.8 & 0 \\
\hline Celiac disease (\%) & 6.2 & \\
\hline
\end{tabular}

continuously on diet, $42.9 \%$ only in case of relapse. $51.2 \%$ thought that relapse can be preceded with diet. Significantly more CD patients believed that lack of diet is a risk factor for both the development and the relapse of IBD ( $p=0.003$ and $p=0.03$ ). $68.6 \%$ of the patients found diet to be as important as medical therapy in the management of IBD. 28\% thought that dietary factors are less important than medications and $2.6 \%$ that it is more important than conservative therapy. Patients obtained dietary recommendations from their physicians in 50\%, from the internet in $48 \%$, from books in $37.7 \%$, and from a dietician in $28.6 \%$ of the cases. $18.2 \%$ of the patients never received any nutritional advice.

The patients described the specific types of foods and drinks triggering or decreasing the activity of the disease. The most "harmful" drinks on disease activity were (in the order of decreasing harmful effect) hard drinks, soda, coffee, beer, wine, fruit drinks, tea, and mineral water. $26.6 \%$ of the patients tried herbs and found them beneficial in $48.8 \%$ of the cases. $85.7 \%$ of the patients were able to indentify foods and ingredients as determinant for relapse. The most frequent ingredients that should be avoided based on the patients' belief: chilli, pepper, curry, and onion. $70.1 \%$ of the patients could identify foods certainly causing symptoms; dairy products, cabbage and spicy foods were the most frequently mentioned. $48.7 \%$ of patients believed that certain foods could prevent disease relapse. Rice, chicken, fish, potatoes, banana, apple and turkey are the most favourable meals with beneficial effect on disease activity.

IBD was associated with lactose intolerance in $14.3 \%$ of the patients (diagnosed with breath test) and with celiac disease (diagnosed previously with duodenal biopsies) in 3.2\%. A history of lactose intolerance or celiac disease were more common in CD than in UC ( $p=0.08 ; p=0.06$ ). $25.3 \%$ of patients regularly eat dairy products, $24.4 \%$ only in remission. $37.7 \%$ developed diarrhoea if eating dairy products. $33.1 \%$ of the patients tried nutrition therapy; $66.7 \%$ of them found it beneficial. The most popular flavour was chocolate and strawberry. More patients liked the liquid than the powder formula.

We asked the patients to list chronic diseases where diet has the most important role. The most frequently chosen chronic disease was diabetes mellitus, followed with celiac disease, CD, UC, and cholelithiasis. We asked whether the patient would agree to jejunal feeding to improve their symptoms. $12 \%$ of the patients would accept this type of therapy, 24\% would refuse it immediately and $74 \%$ would agree to it depending on disease activity.

\section{Discussion}

In this study we examined the patients' perceptions about the benefits and harms of their dietary habits. The questionnaire investigated (i) demographic and clinical information; (ii) patient experience about the association of diet and disease course and (iii) patients' dietary habits and foods that should be avoided to prevent relapse. The most important observations of this study were that half of the patients believed that diet can 
trigger relapse and almost $70 \%$ thought diet to be as important as medical therapy. In the study of Zallot et al. [5] 15.6\% of the patients believed that diet could initiate the disease, while $57.8 \%$ believed that food can play a role in provoking a relapse. Forty percent of the patients identified food as a risk factor off relapse. Using an internet-based questionnaire, Cohen et al. [6] found that yogurt, rice, and banana were more frequently reported to improve symptoms whereas non-leafy vegetables, spicy foods, fruit, nuts, leafy vegetables, fried foods, milk, red meat, soda, popcorn, dairy, alcohol, high-fibre foods, corn, fatty foods, seeds, coffee, and beans were more frequently reported to worsen symptoms. The study of Prince et al. [7] revealed that $62.5 \%$ of the patients felt that food and nutrition were either 'important' or 'extremely important' in their experience of IBD. Patients with CD most frequently reported problems with milk, alcohol, vegetables and high-fibre foods, whereas patients with UC most frequently reported problems with spicy foods, milk, alcohol, fruit and vegetables. In the study of Octoratou et al. [8] the different kinds of food consumption in patients with newly established diagnosis, with previously established diagnosis of $\mathrm{CD}$ and with health controls were assessed. An increased intake of fat, olive oil, margarine, sugar, alcohol drinks, fried food, and pasta was noticed in recently diagnosed patients in comparison with the healthy control group. The list of foods worsening the symptoms of our patients is nearly the same as in the previous papers. However, almost half of the patients could indicate foods seemed to prevent relapse. According to the patiens' observations rice, chicken, fish, potato, banana, apple and turkey might be beneficial in IBD.

New lifestyles include new eating habits in which the consumption of cow's milk also takes precedence [9]. A study examining CD patients from New Zealand revealed that dairy products with a high fat content most frequently worsened CD symptoms [10]. In our study, $25.3 \%$ of the patients can regularly eat dairy products without developing diarrhoea. In a recently published French study, 27.5\% of the patients believed that dairy products may aggravate symptoms during a relapse. However, only $4 \%$ of the respondents adopted a dairy-free diet during a disease flare [5].

Enteral nutrition is often used to correct or to avoid malnutrition which is frequently accompanying IBD. It has also been shown to induce and maintain remission in paediatric patients with CD $[11,12]$. However, the patients' inadequate compliance makes prolonged enteral nutrition difficult to achieve. In our study, only $12 \%$ of the patients reported to certainly agree and more than $70 \%$ of the patients to consider enteral nutrition in case of clinical activity.

Dietary guidance is an important part of the management of patients with IBD. However, in our study, almost $20 \%$ of the patients never received any dietary advice and only in $50 \%$ of the cases were dietary recommendations obtained from physicians. Another study revealed that just under half of all IBD patients reported having seen a dietician as part of their treatment. 33\% of patients who had not seen a dietician stated that they would be interested in attending a support group where they could discuss food and nutrition if it was offered as part of the service. Significantly more CD patients have seen a dietician than those with UC [7]. However, interestingly, the review of Spooren et al. [13] analyzing 41 studies summarized that the current evidence is not sufficient to draw firm conclusions on the role of specific food components or nutrients in the etiology of IBD and the review of Richman et al. [14] revealed little evidence from interventional studies to support specific dietary recommendations in patients with IBD to avoid flare-up.

In our cohort, IBD was presented with diagnosed lactose intolerance in $14.3 \%$ of the patients, mainly in CD. On the basis of genetic polymorphism, breath gases and symptoms, Eadala et al. [15] also confirmed the high proportion of patients with lactose sensitivity in IBD. Lactose intolerance may be more common in IBD than expected; therefore the detection of lactose intolerance and a lactose-free diet in IBD patients may also help to reduce gastrointestinal symptoms.

Diet, due to luminal antigens, seems to have a role in the etiology and pathogenesis of IBD. Identifying dietary factors that are involved in the course and activity of IBD is becoming more and more important in the current management of the disease. Doctors tend to underrate the importance of this factor, whereas most of the patients are sure of its importance in accordance also with some recent published paper. On the basis of our survey, diet seems to be more important in the pathogenesis and management of CD than UC. Most of the patients think that diet can prevent relapse and more than two-thirds of the patients find nutrition to be as important as drug therapy.

\section{Conclusion}

Our results suggest that patients recognize and try to avoid foods they perceive to exacerbate their disease. These results confirm the need of nutritional services for the optimal symptom control as an important part of the therapeutic arsenal of IBD.

\section{Acknowledgement}

This work was supported by the Janos Bolyai Research Scholarship of the Hungarian Academy of Sciences (BO/00632/14/5), TAMOP-4.2.2.A-11/1/KONV-2012-0035, TAMOP-4.2.2-A-11/1/ KONV-2012-0052 TAMOP-4.2.2.A-11/1/KONV-2012-0073 and OTKA PD 105948 (PI: Klaudia Farkas).

\section{References}

1. Zutshi M, Hull TL, Hammel J. Crohn's disease: a patient's perspective. Int J Colorectal Dis. 2007; 22(12): 1437-1444

2. Bernstein CN, Fried M, Krabshuis JH, Cohen H, Eliakim R, Fedail S, et al. World Gastroenterology Organization Practice Guidelines for the Diagnosis and Management of IBD in 2010. Inflamm Bowel Dis. 2010; 16(1): 112-124. doi: 10.1002/ibd.21048.

3. Academy of Nutrition and Dietetics. Available from: http://www. eatright.org/

4. Brown AC, Rampertab SD, Mullin GE. Existing dietary guidelines 
for Crohn's disease and ulcerative colitis. Expert Rev Gastroenterol Hepatol. 2011; 5(3): 411-425. doi: 10.1586/egh.11.29.

5. Zallot C, Quilliot D, Chevaux JB, Peyrin-Biroulet C, Guéant-Rodriguez RM, Freling E, et al. Dietary beliefs and behavior among inflammatory bowel disease patients. Inflamm Bowel Dis. 2013; 19(1): 66-72. doi: 10.1002/ibd.22965.

6. Cohen AB, Lee D, Long MD, Kappelman MD, Martin CF, Sandler RS, et al. Dietary patterns and self-reported associations of diet with symptoms of inflammatory bowel disease. Dig Dis Sci, 2013; 58(5): 1322-1328. doi: 10.1007/s10620-012-2373-3.

7. Prince A, Whelan K, Moosa A, Lomer MC, Reidlinger DP. Nutritional problems in inflammatory bowel disease: The patient perspective. J Crohns Colitis. 2011; 5(5): 443-450. doi: 10.1016/j. crohns.2011.04.016.

8. Octoratou M, Merikas E, Malgarinos G Stanciu C, Triantafillidis JK. A prospective study of pre-illness diet in newly diagnosed patients with Crohn's disease. Rev Med Chir Soc Med Nat Iasi. 2012; 116(1): 40-49.

9. Lucendo AJ, de Rezende LC. Importance of nutrition in inflammatory bowel disease. World J Gastroenterol. 2009; 15(17): 2081-2088.

10. Nolan-Clark D, Tapsell LC, Hu R, Han DY, Ferguson LR. Effects of dairy products on crohn's disease symptoms are influenced by fat content and disease location but not lactose content or disease activity status in a New Zealand population. J Am Diet Assoc. 2011; 111(8): 1165-72. doi: 10.1016/j.jada.2011.05.004.

11. Dupont B, Dupont C, Justum AM, Piquet MA, Reimund JM. Enteral nutrition in adult Crohn's disease: present status and perspectives. Mol Nutr Food Res. 2008; 52(8): 875-884. doi: 10.1002/mnfr.200800093.

12. El-Matary W. Enteral nutrition as a primary therapy of Crohn's disease: the pediatric perspective. Nutr Clin Pract. 2009; 24(1): 9197. doi: $10.1177 / 0884533608329660$.

13. Spooren CE, Pierik MJ, Zeegers MP, Feskens EJ, Masclee AA, Jonkers DM. Review article: the association of diet with onset and relapse in patients with inflammatory bowel disease. Aliment Pharmacol Ther. 2013; 38(10): 1172-1187. doi: 10.1111/apt.12501.

14. Richman E, Rhodes JM. Review article: evidence-based dietary advice for patients with inflammatory bowel disease. Aliment Pharmacol Ther. 2013; 38(10): 1156-1171. doi: 10.1111/apt.12500.

15. Eadala P, Matthews SB, Waud JP, Green JT, Campbell AK. Association of lactose sensitivity with inflammatory bowel disease-demonstrated by analysis of genetic polymorphism, breath gases and symptoms. Aliment Pharmacol Ther. 2011; 34(7): 735-746. doi: 10.1111/j.13652036.2011.04799.x. 